\title{
ELECTROKINETIC PHENOMENA
}

\section{A Small but Constant Source of Error in Measurements OF VISCOSITY \\ By HAROLD A. ABRAMSON \\ (From the Department of Biological Chemistry, College of Physicians and Surgeons, Columbia University)}

(Accepted for publication, November 21, 1931)

During the course of a conversation with Professor Grinnell Jones, we learned of certain inexplicable data obtained during the measurement of the viscosity of solutions of low ionic strength. In particular, variations in the time necessary for a given volume of distilled water to flow out of the (Ostwald type) viscosimeter, as well as certain anomalous features of the viscosity-concentration curves, suggested to us the possibility that some of the difficulties encountered were due to the electric charge on the quartz wall of the viscosimeter. During the laminar flow of a liquid of volume $V$, under hydrostatic pressure $P$, past a quartz surface, a streaming potential $E$, is set up across the capillary of the viscosimeter,

$$
E=\frac{\zeta P D}{4 \pi \eta \kappa}
$$

$\zeta=$ electrokinetic potential or the potential between the surface and the liquid; $D=$ dielectric constant of the medium; $\eta=$ viscosity of the liquid, $\kappa=$ specific conductance of the liquid.* A difference of potential between capillary wall and adjacent solution must exercise a retarding effect on the charged molecules of water near the quartz surface. Thus it must take a longer time for the charged viscosimeter to drain itself than if the wall had been uncharged.

We may calculate as follows the order of magnitude of the error which can be introduced by this retarding effect for the size of capil-

* When the radius of the capillary becomes very small, surface conductance must be considered. 
laries in common use with dilute electrolytes and distilled water. Let $\mathrm{V}_{F}$ be the volume of outflow of the liquid per second from an uncharged capillary of radius $r$. According to Poiseuille,

$$
V_{F}=\frac{\pi P r^{4}}{8 \eta l}
$$

If the wall is charged, a volume $v$ is retarded so that from a charged capillary a volume $\left(V_{F}-v\right)$ will really flow during the time that the volume $V_{F}$ required for outflow. Experience shows that

$$
0<<V_{F}
$$

so that $\frac{v}{V_{F}}=\frac{v}{V_{F}-v}$, very nearly.

The ratio $\frac{v}{V_{F}}$ gives a good idea of the fraction of the total time that is required additionally for the charged capillary. It is approximately correct that the relationship between the streaming potential in the capillary, $E$, and the retarded volume, $v$, is given by the relationship

$$
v=\frac{r^{2} \zeta E D}{t \eta l}
$$

The ratio $\frac{v}{V_{F}}$ is obtained from (2) and (3),

$$
\frac{v}{V_{F}}=\frac{2 \zeta E D}{\pi P} \cdot \frac{1}{r^{2}}=\frac{K}{r^{2}}
$$

where $K$ is a constant.

Kruyt gives the following data for the streaming potentials in a glass capillary with $\mathrm{KCl}$ solutions.

\begin{tabular}{c|c}
\hline $\mathrm{KCl}$ & $E(P=13.6 \mathrm{~cm} . \mathrm{H}, \mathrm{O})$ \\
\hline Mols $\times 10^{0}$ & Volts $\times 10^{3}$ \\
\hline 0 & 350 \\
50 & 102 \\
100 & 57 \\
\hline
\end{tabular}


Since we are concerned with the order of magnitude of a phenomenon in very dilute salt solutions where $E$ and $\zeta$ vary very much from one instance to another, it is sufficient to take a simple illustrative case where $E=200 \times 10^{-3}$ volts and $\zeta=200 \times 10^{-6}$ volts (electrostatic units) to evaluate $K$. The value of $K$ then amounts to $\frac{10^{-9}}{2}$, approximately; $\frac{K}{r^{2}}$ can be obtained from the following table:

\begin{tabular}{c|c|c}
\hline \hline$r$ & $r^{2}$ & $\frac{2 K}{r^{2}}$ \\
\hline$c m$. & $c m^{2}$. & \\
0.041 & 0.031 & $1 \times 10^{1}$ \\
$0.0_{3} 1$ & 0.071 & $1 \times 10^{-1}$ \\
$0.0_{2} 1$ & 0.051 & $1 \times 10^{-3}$ \\
0.01 & $0.0_{8} 1$ & $1 \times 10^{-5}$ \\
0.10 & 0.01 & $1 \times 10^{-7}$ \\
\hline
\end{tabular}

For capillaries $1 \times 10^{-4} \mathrm{~cm}$. in radius the error is as much as $10 \mathrm{per}$ cent. Professor Jones' capillary was of the order $r=0.02 \mathrm{~cm}$. The error due to this effect for capillaries of this radius is of the order $\frac{1}{800,000}$. Professor Jones measures time to one part in 50,000. The use of capillaries less than $r=0.01 \mathrm{~cm}$. should be avoided as the retarding effect then increases most rapidly; thus, for $r=0.01$ it is very near to the limits of experimental error.

Dr. Lars Onsager and Dr. K. C. Cole have been kind enough to discuss this problem in detail with me and to obtain by other methods of calculation the same order of magnitude of the error involved. Dr. Onsager points out that with liquids of very low conductance, the effect is proportional to the square of $\zeta$. This is given in the expression, $\frac{v}{V_{F}}=\frac{8 h^{2} \eta}{\kappa r^{2}}$, where $h=\frac{D \zeta}{4 \pi \eta}$, which Drs. Onsager and Cole have been able to obtain. 